\title{
EL FUTURO DEL CONTROL CONSTITUCIONAL LOCAL: COMENTARIOS A LA CONTRADICCIÓN DE TESIS 350/2009
}

\section{Manlio Fabio CASARÍN LEÓN*}

Con fecha 6 de mayo de 2010, el Pleno de la Suprema Corte de Justicia de la Nación, resolvió la Contradicción de Tesis 350/2009 planteada por la Sala Constitucional del Tribunal Superior de Justicia del Estado de Veracruz, ${ }^{1}$ a partir de los criterios sustentados por el Segundo Tribunal Colegiado en materia Administrativa y el Primer Tribunal Colegiado en materia Administrativa, Segundo Tribunal Colegiado en materia Civil y Tercer Tribunal Colegiado en materia Civil, en cuanto a la procedencia o improcedencia del juicio de amparo directo contra las sentencias dictadas por ella, en los juicios de protección de derechos humanos.

Se trata, a mi juicio, de un asunto relevante para el desarrollo de la justicia constitucional local en los próximos años, pues ha quedado planteado ante nuestro máximo tribunal del país, el alcance de las sentencias pronunciadas por los órganos terminales en materia de control constitucional en el ámbito local.

Como antecedentes del caso, tenemos que el Segundo Tribunal Colegiado en materia Administrativa del Séptimo Circuito, resolvió en esencia lo expuesto por su tesis aislada con registro: 170.900, consultable también en la cuartilla setecientos sesenta y dos, del tomo XXVI de noviembre de dos mil siete, de la Novena Época del Semanario Judicial de la Federación y su Gaceta, de rubro y texto siguientes:

Sentencias dictadas por la Sala Constitucional del Tribunal SUPERIOR DE JuSTICIA DEL ESTAdo DE VERACRUZ. Los TRIBUNALES DE AMPARO CARECEN DE COMPETENCIA PARA CONOCER DE LA IMPUGNACIÓN

* Director de la Facultad de Derecho de la Universidad Veracruzana.

1 El asunto fue remitido a la Corte el 31 de agosto de 2009. 
DE LAS RESOLUCIONES EMITIDAS POR AQUÉLLA AL RESOLVER EL JUICIO DE PROTECCIÓN DE DERECHOS HUMANOS PREVISTO EN LA CONSTITUCIÓN DE DICHA ENTIDAd FEDERATIVA. El Pleno de la Suprema Corte de Justicia de la Nación ha considerado jurisprudencialmente que en el Estado mexicano existen cinco órdenes jurídicos, a saber: el constitucional, el federal, el local o estatal, el del Distrito Federal y el municipal. En cuanto al ámbito estatal se ha desarrollado un fenómeno singular en algunas entidades federativas, como Veracruz, en el sentido de que cuentan con una Sala Constitucional encargada exclusivamente del control de la constitucionalidad local; a ese orden jurídico estatal se le ha denominado teóricamente: Constitucionalismo local. Así, entre los diversos mecanismos jurídicos de control constitucional local en el Estado de Veracruz se encuentra el juicio de protección de derechos humanos, regulado en los artículos 4, 56, fracciones I y II, y 64, fracción I, de su Constitución Política; preceptos que han sido interpretados por el citado Tribunal Pleno en el sentido de que la competencia que la Constitución Local otorga a la Sala Constitucional del Tribunal Superior de Justicia de dicho Estado se circunscribe a conocer y resolver el mencionado juicio de protección de derechos humanos, pero únicamente por cuanto hace a la salvaguarda de los previstos en la Constitución Local. Derivado de esa premisa, estableció una diferencia sustancial entre aquel juicio y el de amparo, consistente en que el primero se limita sólo a proteger derechos humanos que la Constitución de la entidad federativa reserva a sus gobernados, mientras que el juicio de amparo, tutelado en los artículos 103 y 107 de la Constitución Política de los Estados Unidos Mexicanos, comprende la protección de garantías individuales establecidas en el Pacto Federal. Acorde con lo anterior, en las sentencias dictadas por la Sala Constitucional mencionada al conocer del juicio de protección de derechos humanos, el tema de fondo no lo constituye la violación a garantías individuales, sino la relacionada con los derechos humanos previstos en la citada Constitución Local; por ende, los tribunales de amparo carecen de competencia para conocer de la impugnación de dichas sentencias, sin que ello implique una denegación de justicia, pues se trata del reconocimiento y respeto a la autonomía de la Sala Constitucional mencionada para realizar sus funciones como órgano encargado del control de la constitucionalidad local, específicamente en materia de violación a derechos humanos. 
En resumen, la tesis transcrita se funda en las siguientes consideraciones:

a) La interpretación que ha realizado el Pleno de la Corte para determinar que existen cinco órdenes jurídicos en el Estado mexicano: el constitucional, el federal, el local o estatal, el del distrito federal y el municipal.

b) Que Veracruz cuenta con una Sala Constitucional encargada exclusivamente del control de la constitucionalidad local.

c) Que el juicio de protección de derechos humanos consagrado en la Constitución del Estado, faculta a la referida Sala para conocer y resolver el mismo, diferenciándose sustancialmente del juicio de amparo por cuanto a que este último protege las garantías individuales establecidas en la Constitución Federal, mientras que el primero de ellos tutela los derechos humanos que el texto supremo local reserva para sus gobernados.

d) Que con base en lo anterior, los tribunales de amparo carecen de competencia para conocer de la impugnación de las referidas sentencias, sin que ello implique una denegación de justicia, al tratarse del reconocimiento y respeto a la autonomía de la Sala Constitucional como encargada del control de la constitucionalidad local, específicamente en materia de violación a derechos humanos.

Por su parte, el Primer Tribunal Colegiado en materia Administrativa, el Segundo Tribunal Colegiado en materia Civil, así como el Tercer Tribunal Colegiado en materia Civil, todos del Séptimo Circuito, decidieron declarar procedente el juicio de amparo directo en contra de las sentencias dictadas por la Sala Constitucional en el juicio de protección de derechos humanos local, con independencia de que en el fondo del asunto negaron la protección de la justicia federal. ${ }^{2}$

La tesis transcrita es relevante en el sentido de que se trata de un intento por favorecer el desarrollo de los sistemas locales de control constitucional, atendiendo al criterio de que las sentencias dictadas por tribunales, cortes o salas constitucionales, dada la naturaleza fundamental de los conflictos que dirimen, poseen características, modalidades y matices 
propios, distintos de aquellas resoluciones dictadas por los órganos de la jurisdicción ordinaria.

En efecto, las resoluciones dictadas por los órganos de control constitucional, a diferencia de aquellas emitidas por la jurisdicción ordinaria, cuentan al menos con las siguientes características:

a) Se trata de sentencias dictadas por órganos terminales o de cierre del sistema, es decir, aquellos que cuentan con la facultad de interpretación final de la Constitución y el monopolio de rechazo normativo.

b) Son definitivas e inatacables (poseen autoridad de cosa juzgada).

c) Sus efectos trascienden a las partes en la controversia (implicaciones políticas).

d) Generan precedentes obligatorios (jurisprudencia) para los órganos jurisdiccionales, administrativos y legislativos del Estado. $^{3}$

No obstante lo anterior, para el asunto que nos ocupa y a reserva de comentar más adelante las principales razones bajo los cuales se sustentó la resolución de la Corte en la contradicción de tesis planteada, tenemos que resulta discutible lo argumentado por el Tribunal Colegiado en la tesis transcrita, así como el resultado al que llega, por varios aspectos.

En primer lugar, si bien ha existido en el pasado reciente un pronunciamiento mayoritario por parte de los ministros de la Suprema Corte de Justicia, por cuanto hace al aval otorgado a la Sala Constitucional de Veracruz para conocer del juicio de protección de derechos humanos, ${ }^{4}$

3 Cfr. La Ponencia General de mi autoría intitulada "El desarrollo de los sistemas locales de control constitucional a partir de su articulación con el sistema federal: análisis de la experiencia veracruzana”, presentada en el X Congreso Nacional de Derecho Constitucional de los Estados, celebrado en diciembre de 2009 en San Cristóbal de las Casas, Chiapas, y contenida en la Memoria in Extenso del evento, próxima a ser publicada por el Instituto de Investigaciones Jurídicas de la UNAM.

4 El 9 de mayo de 2002, la Suprema Corte de Justicia resolvió diversas controversias constitucionales presentadas por los Ayuntamientos de Xalapa (10/2000), La Antigua (15/2000), Córdoba (16/2000), Tomatlán (17/2000) y San Juan Rodríguez Clara (18/2000), los que demandaron entre otras cuestiones, la invalidez del decreto de reforma integral a la Constitución veracruzana promulgada en febrero de 2000, al estimar que con la creación del juicio para la protección de derechos humanos, cuya competencia se le atribuye a la Sala Constitucional del Tribunal Superior de Justicia de dicha entidad, se 
mismo que quedó asentado en la tesis aislada XXXIII/2002, ${ }^{5}$ no menos importante resulta el análisis del voto de minoría ${ }^{6}$ derivado de dicho asunto, el cual arriba a la conclusión de que, haciendo una comparación minuciosa, existe identidad material entre los derechos consagrados en la Constitución federal bajo el nombre de garantías individuales y aquellos consagrados en la Constitución veracruzana, ya sea que se interpreten

invadía la esfera competencial de los Tribunales de la Federación, específicamente por lo que hace al juicio de amparo.

5 Dicho criterio del Pleno de la Suprema Corte de Justicia quedó reflejado en la tesis XXXIII/2002, que a la letra dispone: "CONTROVERSIA CONSTITUCIONAL. LA FACULTAD otorgada a la Sala CONSTitucional del Tribunal Superior De Justicia DEL EsTADO DE VERACRUZ-LLAVE PARA CONOCER Y RESOLVER EL JUICIO DE PROTECCIÓN DE DERECHOS HUMANOS PREVISTO EN LA CONSTITUCIÓN POLÍTICA DE ESA ENTIDAD FEDERATIVA, NO INVADE LA ESFERA DE ATRIBUCIONES DE LOS TRIBUNALES DE LA FEDERACIÓN, PUES AQUÉL SE LIMITA A SALVAGUARDAR, EXCLUSIVAMENTE, LOS DERECHOS HUMANOS QUE ESTABLECE EL PROPIO ORDENAMIENTO LOCAL. De la interpretación armónica y sistemática de lo dispuesto en los artículos 4, párrafo tercero, 56, fracciones I y II y 64, fracción I, de la Constitución Política del Estado de Veracruz-Llave, así como de la exposición de motivos del decreto que aprobó la Ley 53 mediante la cual aquéllos fueron reformados, se desprende que la competencia que la Constitución local le otorga a la Sala Constitucional del Tribunal Superior de Justicia del Estado de Veracruz-Llave, se circunscribe a conocer y resolver el juicio para la protección de derechos humanos, pero únicamente por cuanto hace a la salvaguarda de las previstos en la Constitución de aquella entidad federativa, por lo que dicha Sala no cuenta con facultades para pronunciarse sobre violaciones a las garantías individuales que establece la Constitución Política de los Estados Unidos Mexicanos. Acorde con lo anterior, se concluye que los preceptos citados no invaden las atribuciones de los tribunales de la Federación, en tanto que el instrumento para salvaguardar los derechos humanos que prevé la Constitución local citada, se limita exclusivamente a proteger los derechos humanos que dicha Constitución reserve a los gobernados de esa entidad federativa; mientras que el juicio de amparo, consagrado en los artículos 103 y 107 de la propia Constitución federal, comprende la protección de las garantías individuales establecidas en la parte dogmática del pacto federal, de manera que la mencionada Sala Constitucional carece de competencia para resolver sobre el apego de actos de autoridad a la carta magna. Lo anterior se corrobora si se toma en consideración que el instrumento jurídico local difiere del juicio de garantías en cuanto a su finalidad, ya que prevé que la violación de los derechos humanos que expresamente se reservan implicará la sanción correspondiente y, en su caso, la reparación del daño conforme lo dispone el artículo 4o. de la propia Constitución estatal, lo que no acontece en el indicado mecanismo federal", Pleno, Semanario Judicial de la Federación y su Gaceta XVI, Novena Época, agosto de 2002, p. 903, tesis aislada, P. XXXIII/2002.

6 Sentencia y voto de minoría pronunciados en la Controversia Constitucional 16/2000, publicados en el DOF el 21 de junio de 2002. 
literal, doctrinal o jurisprudencialmente. Por ello, el medio de control constitucional idóneo es el amparo.

En segundo lugar, no es exacta la afirmación contenida en la tesis que se comenta, cuando afirma que el estado de Veracruz cuenta con una Sala Constitucional encargada exclusivamente del control de la constitucionalidad local, pues de acuerdo al diseño institucional en vigor, dicha atribución la comparte con el Pleno del Tribunal Superior de Justicia, quien si bien no tiene intervención en el juicio de protección de derechos humanos, se constituye en órgano terminal en materia de controversias constitucionales, acciones de inconstitucionalidad y acciones por omisión legislativa locales. ${ }^{7}$

En tercer lugar, arribar a la conclusión de que los tribunales de amparo carecen de competencia para conocer de la impugnación de las sentencias en materia de control constitucional local, tomando como referencia las anteriores consideraciones, inclusive afirmando que no se trata de una denegación de justicia sino del reconocimiento y respeto a la autonomía de la Sala Constitucional como encargada del referido control, es desconocer la estructura constitucional, legal y jurisprudencial bajo la cual opera actualmente el juicio de amparo, incluyendo sus efectos jurídico-procesales relacionados con la tutela efectiva de los derechos fundamentales.

A mayor abundamiento, el Segundo Tribunal Colegiado en materia Administrativa del Séptimo Circuito intenta introducir vía jurisprudencia una causal de improcedencia no prevista en el artículo 73 de la Ley de Amparo, ${ }^{8}$ aunado a que de acuerdo con las reglas para el ejercicio del

7 Artículos. 56, fracciones I y II, 64 y 65 de la Constitución Política del Estado de Veracruz de Ignacio de la Llave.

${ }^{8}$ No obstante que el referido precepto señala de manera expresa en su fracción XVIII, la existencia de otros casos de improcedencia no establecidos en el mismo, aquélla deberá derivar indefectiblemente de alguna disposición de la propia Ley de Amparo o de la Constitución federal. Véase la tesis "IMPROCEDENCIA. INTERPRETACIÓN DE LA FRACCIÓN XVIII DEL ARTíCULO 73 DE LA LEY DE AMPARO”. Esta fracción debe interpretarse en el sentido de que las causas de improcedencia del juicio de garantías que en forma enunciativa prevé, deben derivar necesariamente de cualquier mandamiento de la propia Ley de Amparo o de la carta magna, lo que de suyo implica que las diecisiete primeras fracciones del artículo 73 de la Ley de Amparo sólo establecen algunos de los supuestos de improcedencia del juicio de amparo, pero esos supuestos no son los únicos en que dicho juicio puede estimarse improcedente, pues existen otras causas claramente previstas en algunos de los preceptos de la Constitución Federal y de la propia Ley Reglamentaria 
juicio de garantías en la vía directa, sigue siendo procedente la impugnación de sentencias o resoluciones definitivas de tribunales locales por violación a la garantía de legalidad prevista en los artículos 14 y 16 de la Constitución federal. ${ }^{9}$

Asimismo, derivado del carácter uniinstancial del juicio de protección de derechos humanos, decir que la resolución que se adopte en el mismo tendrá la calidad de cosa juzgada (sin posibilidad de contar con una instancia de revisión), implica contravenir derechos consagrados en pactos e instrumentos internacionales de los cuales forma parte nuestro país y que se encuentran incorporados a nuestro ordenamiento jurídico. Derechos relacionados con las garantías judiciales en general, y en particular con el acceso a la jurisdicción, debido proceso y tutela judicial efectiva. ${ }^{10}$

de los artículos 103 y 107 Constitucionales. Por tanto, no es exacto que exista imprecisión en torno de las causas de improcedencia que se prevén en esa fracción. Amparo en revisión 475/99. Saúl Ortiz Cruz, 12 de mayo de 1999, unanimidad de cuatro votos, ausente: José Vicente Aguinaco Alemán. Ponente: Mariano Azuela Güitrón, secretaria: Irma Rodríguez Franco, tesis: 2a. LXXXVI/99, tesis aislada, materia(s): común, Segunda Sala, Semanario Judicial de la Federación y su Gaceta IX, junio de 1999, Novena Época, p. 373.

9 En efecto, el artículo 158 de la Ley de Amparo señala: "El juicio de amparo directo es competencia del Tribunal Colegiado de Circuito que corresponda, en los términos establecidos por las fracciones V y VI del artículo 107 constitucional, y procede contra sentencias definitivas o laudos y resoluciones que pongan fin al juicio, dictados por tribunales judiciales, administrativos o del trabajo, respecto de los cuales no proceda ningún recurso ordinario por el que puedan ser modificados o revocados, ya sea que la violación se cometa en ellos o que, cometida durante el procedimiento, afecte a las defensas del quejoso, trascendiendo al resultado del fallo, y por violaciones de garantías cometidas en las propias sentencias, laudos o resoluciones indicados. Para los efectos de este artículo, sólo será procedente el juicio de amparo directo contra sentencias definitivas o laudos y resoluciones que pongan fin al juicio, dictados por tribunales civiles, administrativos o del trabajo, cuando sean contrarios a la letra de la ley aplicable al caso, a su interpretación jurídica o a sus principios generales de Derecho a falta de ley aplicable, cuando comprendan acciones, excepciones o cosas que no hayan sido objeto del juicio, o cuando no las comprendan todas, por omisión o negación expresa.

10 Recordemos el texto de los artículo 8, 2 h) y 25 de la Convención Americana de Derecho Humanos firmada en San José, Costa Rica en 1969, que a la letra disponen: "Artículo 8. Garantías Judiciales. 1. Toda persona tiene derecho a ser oída con las debidas garantías y dentro de un plazo razonable, por un juez o tribunal competente independiente e imparcial establecido con anterioridad por la ley, en la sustanciación de cualquier acusación penal formulada contra ella, o para la determinación de sus derechos y obligaciones de orden civil, laboral, fiscal o de cualquier otro carácter. 2... Durante el proceso, toda persona tiene derecho, en plena igualdad, a las siguientes garantías mínimas:...h) derecho 
En este orden de ideas, gran parte de los argumentos esgrimidos fueron determinantes para que el Pleno de la Suprema Corte de Justicia de la Nación, resolviera por una mayoría de diez votos contra uno, la contradicción de tesis 350/2009 en la sesión pública del 6 de mayo de 2010, ${ }^{11}$ en el sentido de que debe prevalecer el criterio de la procedencia del amparo directo ante los tribunales colegiados de circuito en contra de las sentencias dictadas por la Sala Constitucional de Veracruz en el juicio de protección de derechos humanos.

Es importante destacar, que en las sesiones públicas del 4 y 6 de mayo de 2010, la ministra ponente Margarita Beatriz Luna Ramos, sometió al Pleno la propuesta de resolución en el sentido de que efectivamente existía la contradicción de tesis entre los tribunales colegiados referidos, y que era procedente el juicio de amparo directo en relación con las sentencias dictadas por la Sala Constitucional de Veracruz, básicamente por considerar que se trata de una resolución jurisdiccional de una Sala que integra el Tribunal Superior de Justicia del Estado, y que por tanto se encuentra dentro de los supuestos del artículo 158 de la Ley de Amparo, referidos a la procedencia del amparo directo.

Dentro de los múltiples e interesantes argumentos manifestados por los miembros de nuestro Máximo Tribunal, destacan aquellos realizados por el ministro Arturo Zaldívar Lelo de Larrea, quien secundando el proyecto a discusión expuso que: 1) este tipo de tribunales o de organismos locales están sujetos a control constitucional de amparo, porque para no estarlo se requeriría texto expreso en la Constitución general; 2) que si se está en presencia de la resolución de un Tribunal como era el caso, la denominación de tribunales judiciales, administrativos y del trabajo contempladas en la ley de amparo, encuadra perfectamente en las hipótesis para su procedencia, y 3) que en la práctica el amparo directo viene a ser

de recurrir del fallo ante juez o tribunal superior"; artículo 25. Protección Judicial. 1. Toda persona tiene derecho a un recurso sencillo y rápido o a cualquier otro recurso efectivo ante los jueces o tribunales competentes, que la ampare contra actos que violen sus derechos fundamentales reconocidos por la Constitución, la ley o la presente convención, aún cuando tal violación sea cometida por personas que actúen en ejercicio de sus funciones oficiales. 2. Los Estados partes se comprometen: b) a desarrollar las posibilidades de recurso judicial...". Para el caso que nos ocupa, se cuestiona que no exista como mínimo un recurso de revisión ante el Pleno del Tribunal Superior de Justicia.

11 Véanse las versiones taquigráficas de las sesiones públicas del 4 y 6 de mayo de 2010, en www.scjn.gob.mx. 
una especie de recurso lato sensu, en donde se analiza la legalidad de la resolución, so pretexto de violación de las garantías establecidas en los artículos 14 y 16 de la Constitución federal.

De igual manera, el ministro José Ramón Cossío manifestó — esencialmente - en sus diversas intervenciones, que: 1) las sentencias locales en materia de derechos humanos no podrían válidamente afectar el contenido esencial de las garantías individuales reconocidas en la norma suprema, si se toma en cuenta que el orden jurídico local está supeditado al orden jurídico constitucional; 2) lo que busca garantizarse a través del juicio de amparo directo es que los tribunales colegiados, lejos de actuar como jueces del orden jurídico federal, funcionan como jueces de la Constitución, con excepción de la materia electoral, y 3) que los derechos que se hicieron valer ante el Tribunal Superior de Justicia de Veracruz para que conozca de un juicio de constitucionalidad local terminan con una sentencia definitiva, razón por la cual los quejosos pueden plantear la violación a un derecho fundamental contenido en la Constitución Federal, misma que, sin excepción, no puede ser más que conocida por los Tribunales del Poder Judicial de la Federación, en cuanto órganos encargados de la tutela del orden constitucional total.

Por su parte, el ministro Sergio Valls Hernández expuso diferentes argumentos para discrepar del sentido del proyecto, básicamente a partir de las siguientes razones: 1) si el referido juicio constitucional local tiene por objeto conocer de violaciones a los derechos humanos establecidos en la Constitución de Veracruz, ello debe entenderse como una instancia definitiva dentro de ese sistema de justicia constitucional, pues su objeto se limita a garantizar su propio orden jurídico; 2) estimar que en contra de las sentencias dictadas por la Sala Constitucional de la entidad procede un amparo legalidad como es el amparo directo, implica romper con la justicia constitucional local al someterla siempre al escrutinio de los tribunales federales, y 3) sostener que por medio de este proceso, los tribunales colegiados y la Suprema Corte van a garantizar que la interpretación de los derechos humanos del orden jurídico local no afecte el contenido esencial de las garantías individuales del orden jurídico constitucional al que se encuentran supeditados es tanto como romper con el federalismo, según el cual las entidades federativas se dan sus propias Constituciones, y por tanto, sus propias garantías procesales para salvaguardarlas. 
Como hemos podido observar, se trata de una resolución relevante en donde la Corte ha determinado - por mayoría de votos - la procedencia del amparo directo ante los tribunales colegiados de circuito en contra de las sentencias dictadas por la Sala Constitucional de Veracruz. Sin embargo, me parece oportuno referirme al tema de fondo con que, me parece, debe resolverse a futuro el tema en comento.

En un Estado federal como el nuestro, tratándose de mecanismos de protección de derechos fundamentales consagrados en las entidades federativas, resulta imprescindible explorar las posibilidades en torno al desarrollo de un recurso judicial local; esto es, podríamos pensar en que el conocimiento de los asuntos podría ser confiado en primera instancia a los órganos jurisdiccionales ordinarios (amparo judicial) y en revisión (recurso de amparo constitucional) a la Sala Constitucional; o una segunda posibilidad estaría determinada por el conocimiento originario de los asuntos a cargo de la referida Sala y la procedencia de un recurso de revisión ante el Pleno del Tribunal Superior de Justicia.

En aras de fortalecer el desarrollo y eficacia, no nada más de los instrumentos de amparo locales vigentes y aquellos que en el futuro se llegaren a consagrar por el resto de las entidades federativas, sino atendiendo al conjunto de mecanismos de justicia constitucional en los estados de la República, ${ }^{12}$ me parece que limitar por la vía jurisprudencial la procedencia del amparo u otro mecanismo federal por parte del Poder Judicial de la Federación para favorecer el desarrollo de los sistemas de control constitucional locales, o incluso disponer en el ámbito local limitaciones a la jurisdicción federal sin contar con un adecuado diseño normativoinstitucional, además de contravenir el espíritu de la Constitución federal, conlleva en la actualidad enormes riesgos en la esfera de temas tan delicados como la protección de derechos humanos. De ahí la importancia de que la Corte haya resuelto la contradicción de tesis en los términos apuntados.

${ }_{12}$ Para el caso de las controversias constitucionales, acciones de inconstitucionalidad y acciones por omisión legislativa locales, resulta razonable el fallo de un tribunal, corte o sala constitucional en única instancia. Sin embargo, a mi juicio, tratándose de mecanismos de protección de derechos fundamentales, sería deseable que las entidades federativas diseñen cuando menos dos instancias, que bien podrían ser los órganos jurisdiccionales ordinarios (amparo judicial) y en revisión (recurso de amparo constitucional) en donde la competencia se surtiría en favor de los órganos terminales. 
Por ello, estimamos que la mejor manera de articular los sistemas de defensa constitucional federal y local, es a partir de la reforma al artículo 116 de la Constitución general, ${ }^{13}$ de tal suerte que se establezcan las bases y principios esenciales para que los Estados diseñen mecanismos de control constitucional local de manera integral y uniforme, con una magistratura constitucional especializada, con la determinación de los sujetos legitimados para acudir a ella, con la determinación del parámetro de control ejercido, los supuestos de definitividad y efectos de las sentencias, los alcances interpretativos de la jurisprudencia local, así como los contenidos que necesariamente deberán desarrollar las constituciones y el legislador ordinario local en la confección de las normas del proceso constitucional. ${ }^{14}$

13 Obviamente para que pudiera operar eficazmente el diseño propuesto, tendríamos que revisar y armonizar otros preceptos constitucionales, tales como el 103, 104, 105, 107, 124 y 133, entre otros, contemplando la posibilidad de que la Suprema Corte de Justicia pueda ejercer su facultad de atracción para conocer de asuntos relevantes de la jurisdicción constitucional local, básicamente cuando se trate de violaciones directas a la carta marga federal.

14 Entre los pocos intentos por reformar el artículo 116 de la Constitución general de la República para introducir las bases del control constitucional local, destaca la propuesta del senador Renán Cleominio Zoreda Novelo, del grupo parlamentario del Partido Revolucionario Institucional, cuya iniciativa y exposición de motivos fueron presentados al Senado en el mes de marzo de 2007, turnándose para su estudio a las Comisiones Unidas de Puntos Constitucionales y de Estudios Legislativos. La reforma que se propone, asimismo, podría contemplar en los artículos transitorios del decreto respectivo un plazo perentorio para que las entidades federativas armonicen sus ordenamientos, tal y como ha sucedido recientemente con la reformas constitucionales electoral en 2007 y en materia de justicia penal en 2008 . 